\title{
27.
}

\section{SUR LES ACTIONS MUTUELLES DES FORMES INVARIANTIVES DÉRIVÉES.}

\author{
[Crelle's Journal, Lxxxv. (1878), pp. 89-114.]
}

JE comprends les invariants, les covariants, les contravariants et toutes les formes qui dérivent dans le même sens d'un système donné de Quantics sous le nom général de dérivées invariantives, et je vais établir un principe qui rend ces formes fécondes et donne à deux quelconques d'entre elles la faculté de produire, par l'action de l'une sur l'autre, de nouvelles formes invariantives. Si l'on se borne aux invariants d'un seul Quantic ou d'un système de Quantics, la manière de procéder pour cette génération est presque évidente d'elle-même. Car soient $F(a, b, c, \ldots), G(a, b, c, \ldots)$ deux invariants du même Quantic, ou du même système de Quantics, et écrivons à la place de $a, b, c, \ldots$ dans l'une de ces deux fonctions $\frac{d}{d a}, \frac{d}{d b}, \frac{d}{d c}, \ldots$ : si l'on opère avec la fonction ainsi modifiée sur l'autre, le résultat restera invariantif (sauf le cas dans lequel le résultat se réduit à zéro ou à une autre constante numérique). En effet, désignons par $\dot{a}, \dot{b}, \ldots$ les opérations $\frac{d}{d a}, \frac{d}{d b}, \ldots$ et admettons qu'une substitution quelconque appliquée aux variables des Quantics donnés dont $F$ et $G$ sont les dérivées, change en $a^{\prime}, b^{\prime}, c^{\prime}, \ldots$ les éléments donnés $a, b, c, \ldots$; alors on aura d'après un principe élémentaire du calcul différentiel

$$
\begin{aligned}
& \dot{a}^{\prime}=\dot{a} \frac{d a}{d a^{\prime}}+\dot{b} \frac{d b}{d a^{\prime}}+\ldots \\
& \dot{b}^{\prime}=\dot{a} \frac{d a}{d b^{\prime}}+\dot{b} \frac{d b}{d b^{\prime}}+\ldots
\end{aligned}
$$

donc la substitution contraire à la substitution en question changera $\dot{a}, \dot{b}, \dot{c}, \ldots$ en $\dot{a}^{\prime}, \dot{b}^{\prime}, \dot{c}^{\prime}, \ldots$ Or $F(\dot{a}, \dot{b}, \dot{c}, \ldots)$ et $G(a, b, c, \ldots)$ ne subissant aucun changement, le résultat de l'opération de la première sur la seconde ne subira non plus de changement par une substitution quelconque opérée sur les variables.

Ce raisonnement reste bon dans le cas où l'on substitue à l'invariant $G$ un contravariant quelconque. Mais dans le cas général, dans lequel les variables entrent en même temps dans $F$ et dans $G$, on a besoin de s'appuyer sur des considérations additionnelles d'un genre nouveau. 


\section{7] Les actions mutuelles des formes invariantives dérivées}

Or je remarque que la formation d'un Quantic quelconque se compose de trois genres des quantités-des variables,-des parties littérales des coefficients et enfin-des multiplicateurs numériques qui les affectent et qui forment, pour ainsi dire, l'équipement arithmétique de la forme. Dans la vieille algèbre ces multiplicateurs numériques ont été réduits à l'unité, dans l'algèbre moderne on les égale aux nombres binômes ou polynômes. Dans la théorie que je vais produire on aura besoin de se servir d'un équipement qui tient pour ainsi dire la moyenne entre les deux dont je viens de parler, c. à d. que le multiplicateur d'un élément quelconque sera la racine carrée du nombre binôme ou polynôme qui lui serait égalé dans la notation ordinaire des Quantics. Quand les multiplicateurs numériques sont mis sous cette forme, je dirai que le Quantic est un Quantic préparé.

Remarquons que, quel que soit l'équipement numérique d'un Quantic, une substitution quelconque opérée sur les variables induit une substitution corrélative opérée sur les éléments, c. à d. que si dans le Quantic $(\lambda a, \mu b, \nu c, \ldots \chi x, y, z, \ldots)^{i}$ on écrit pour $x, y, z, \ldots$ des fonctions linéaires de $x, y, z, \ldots$, le Quantic se changera en un autre $(\lambda A, \mu B, \nu C, \ldots \chi \chi x, y, z, \ldots)^{i}$ où $A, B, C, \ldots$ seront des fonctions linéaires de $a, b, c, \ldots$ Qu'on sépare les coefficients qui entrent dans les fonctions linéaires données de $x, y, z, \ldots:$ on obtient une matrice;-qu'on sépare les coefficients qui entrent dans les fonctions linéaires de $a, b, c, \ldots$ : on aura une autre matrice,-et je dirai que cette seconde matrice est induite par la première. Puisque les changements peuvent être effectués par des altérations insensibles on pourrait même sans trop d'incorrection affirmer que le mouvement des variables induit ou entraîne avec lui un mouvement dans les éléments d'une forme donnée. L'ordre du déterminant inducteur ne dépend que du nombre des variables, celui du déterminant induit dépend en même temps du nombre des variables et du degré de la forme par rapport aux variables.

On comprend le sens qu'il faut attribuer à la désignation de matrices contraires. La matrice dont les termes servent à exprimer le système des variables $\xi, \eta, \zeta, \ldots$ en fonctions linéaires des variables $x, y, z, \ldots$ et celle dont les termes servent à exprimer les $x, y, z, \ldots$ en fonctions linéaires $\operatorname{des} \xi, \eta, \zeta, \ldots$ seront appelées matrices contraires*, et de même les deux substitutions dont les coefficients sont données par deux matrices contraires seront appelées substitutions contraires.

Cela posé, je suis en état d'énoncer le suivant théorème fondamental. Dans un Quantic préparé deux substitutions contraires opérées sur les variables induisent deux substitutions contraires opérées sur les éléments. La préparation indiquée ci-dessus est la condition nécessaire pour la validité

* Mot plus précis que celui d'inverse ou de réciproque dont on se sert quelquefois dans un ens plus vague quant à la grandeur absolue des termes dont on parle. 
du théorème: si le Quantic n'était pas dûment préparé de la manière indiquée, des substitutions contraires appliquées aux variables n'induiraient point des substitutions contraires opérées sur les éléments. Avant d'entrer dans la démonstration de ce théorème je rappellerai que la matrice produite par la multiplication de deux et conséquemment d'un nombre quelconque de matrices et la matrice produite par la multiplication des matrices respectivement contraires aux premières, sont encore des matrices contraires entre elles-cet énoncé suppose toutefois que les multiplications des matrices soient effectuées de manière que leurs éléments soient combinés ligne par colonne ou colonne par ligne, mais non ligne par ligne ou colonne par colonne.

Considérons pour un instant une matrice de la forme

$$
\begin{array}{llll}
1 & r^{\prime} & & \\
r & 1 & s^{\prime} & \\
& s & 1 & t^{\prime} \\
& & t & 1
\end{array} .
$$

où toutes les places vacantes dans le carré dont la ligne des unités est une des diagonales, doivent être remplies par des zéros.

En se bornant aux termes de la première dimension en $r, s, t, \ldots$, la valeur du déterminant est $1-r r^{\prime}-s s^{\prime}-t t^{\prime} \ldots$. Autant qu'il est permis de se servir de cette valeur approchée, les dérivées du déterminant par rapport à $r, s, t, \ldots$ auront les valeurs $-r^{\prime},-s^{\prime},-t^{\prime}, \ldots$ et vice versâ, donc en attribuant des valeurs infiniment petites à $r, s, t, \ldots$ et faisant évanouir chacune des lettres accentuées, la matrice

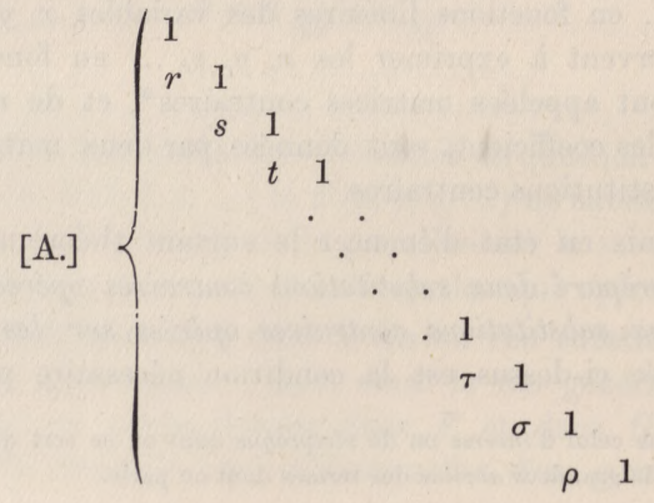


aura pour sa contraire la matrice

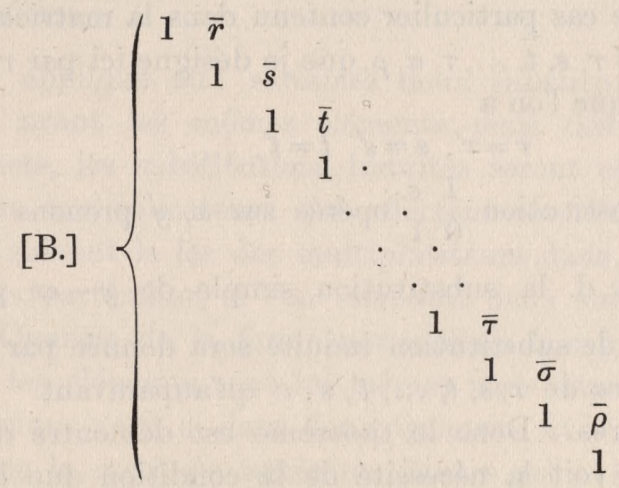

où $\bar{r}, \bar{s}, \bar{t}, \ldots$ doivent être remplacées par $-r,-s,-t, \ldots$.

J'établirai d'abord la loi de l'induction des contraires dans le cas d'un Quantic binôme dûment préparé, $x$ et $y$ étant les variables et $a, b, c, \ldots$ les éléments.

Si la loi des contraires est vraie pour des substitutions dont la matrice a l'unité pour valeur de son déterminant, elle sera vraie pour des substitutions quelconques. De plus on démontre aisément que toute substitution au déterminant $=1$ opérée sur $x, y$ peut être effectuée par trois substitutions simples successives, c. à d. par les substitutions successives de $x+h y$ pour $x$, de $y+k x$ pour $y$ et de $x+l y$ pour $x$. Donc en vertu d'une remarque faite précédemment, la démonstration cherchée se réduit à la démonstration dans le cas d'une substitution simple, c. à d. de la substitution de $x+h y$ pour $x$. Mais cette substitution même peut être effectuée par une succession infinie de substitutions de la forme $x+\epsilon y$, où $\epsilon$ est une quantité infiniment petite: donc en vertu de la même remarque il ne nous reste qu'à établir la loi des contraires dans le cas où l'on substitue $x+\epsilon y$ au lieu de $x$ dans la forme préparée

$$
\begin{aligned}
a x^{i}+\sqrt{ }(i) b x^{i-1} y+\sqrt{ }\left\{\frac{i(i-1)}{2}\right\} & c x^{i-2} y^{2}+\ldots \\
+ & \sqrt{ }\left\{\frac{i(i-1)}{2}\right\} h x^{2} y^{i-2}+\sqrt{ }(i) k x y^{i-1}+l y^{i}
\end{aligned}
$$

Soit

$$
a^{\prime} x^{i}+\sqrt{ }(i) b^{\prime} x^{i-1} y+\ldots+\sqrt{ }(i) k^{\prime} x y^{i-1}+l^{\prime} y^{i}
$$

ce que devient la forme après cette substitution, de sorte que

$$
\begin{aligned}
a^{\prime}=a, b^{\prime}=b+\sqrt{ }(i) \epsilon a, c^{\prime} & =c+\sqrt{ }\{2(i-1)\} \epsilon b, \ldots, \\
k^{\prime} & =k+\sqrt{ }\{2(i-1)\} \epsilon h, l^{\prime}=l+\sqrt{ }(i) \epsilon k,
\end{aligned}
$$

et posons

$\sqrt{ }(i) \epsilon=r, \sqrt{ }\{2(i-1)\} \epsilon=s, \sqrt{ }\{3(i-2)\} \epsilon=t, \ldots, \sqrt{ }\{2(i-1)\} \epsilon=s^{\prime}, \sqrt{ }(i) \epsilon=r^{\prime}$, 
la matrice de la substitution par laquelle $a, b, c, \ldots$ se transforment en $a^{\prime}, b^{\prime}, c^{\prime}, \ldots$ formera le cas particulier contenu dans la matrice [A.] lorsque le système des quantités $r, s, t, \ldots, \tau, \sigma, \rho$, que je désigne ici par $r, s, t, \ldots, t^{\prime}, s^{\prime}, r^{\prime}$, est réversible, c. à d. que l'on a

$$
r=r^{\prime}, s=s^{\prime}, t=t^{\prime}, \ldots
$$

Or au lieu de la substitution $\begin{array}{ll}1 & \epsilon \\ 0 & 1\end{array}$ opérée sur $x, y$ prenons la substitution contraire $\begin{array}{r}10 \\ -\epsilon\end{array}$, c. à d. la substitution simple de $y-\epsilon x$ pour $y$ : il est évident que la forme de substitution induite sera donnée par la matrice [B.] avec les mêmes valeurs de $r, s, t, \ldots, t^{\prime}, s^{\prime}, r^{\prime}$ qu'auparavant. Mais ces deux matrices sont contraires. Donc le théorème est démontré dans le cas des formes binaires. On voit la nécessité de la condition que le Quantic soit préparé quant à son équipement numérique. Car sans cela les deux matrices induites qui se trouvent toujours sous les deux formes [A.] et [B.] ne seraient plus contraires, car le système des quantités $r, s, t, \ldots, t^{\prime}, s^{\prime}, r^{\prime}$ étant renversé dans ces deux formes et les lettres accentuées et non-accentuées ne conservant plus des valeurs identiques, les deux matrices [A.] et [B.] cesseraient d'être corrélatives.

Comme exemple du théorème qui vient d'être établi, considérons le cas très-simple de la forme préparée $a x^{2}+\sqrt{ }(2) b x y+c y^{2}$.

Opérons sur $x, y$ la substitution $f x+g y$ pour $x$ et $h x+k y$ pour $y$ (où pour plus de simplicité je supposerai que $f k-g h=1)$; les valeurs induites en $a, b, c$ répondront à la matrice

$\begin{array}{ccc}f^{2}, & \sqrt{ }(2) f h, & h^{2}, \\ \sqrt{ }(2) f g, & f k+g h, & \sqrt{ }(2) h k, \\ g^{2}, & \sqrt{ }(2) g k, & k^{2},\end{array}$

dont l'inverse, en négligeant le facteur commun $f k-g h$, sera

$$
\begin{array}{ccc}
k^{2}, & -\sqrt{ }(2) g k, & g^{2}, \\
-\sqrt{ }(2) k h, & g h+f k, & -\sqrt{ }(2) f g, \\
h^{2}, & -\sqrt{ }(2) f h, & f^{2},
\end{array}
$$

qui est évidemment la matrice d'induction qui répond à la substitution de $k x-h y$ pour $x$ et de $-g x+f y$ pour $y$ : c. à d. que les deux substitutions contraires $\begin{aligned} & f g, \quad k-h \\ & h k\end{aligned}{ }^{\prime}-g \quad f$, opérées sur les variables induisent des substitutions contraires opérées sur les éléments $a, b, c$.

J'ajouterai deux observations dont la première trouvera son application dans la démonstration générale et dont la seconde facilitera l'application du principe que je vais fonder sur la loi des contraires.

$1^{\circ}$. Il est évident que pour préparer un Quantic, il n'est pas nécessaire que les multiplicateurs numériques soient les nombres binômes eux-mêmes; 
il suffit que les rapports entre ces multiplicateurs soient les mêmes qu'entre les nombres binômes.

$2^{\circ}$. Si l'on applique aux variables deux substitutions contraires dans deux Quantics ayant les mêmes éléments mais des multiplicateurs numériques distincts, les substitutions induites seront contraires pourvu que les produits des multiplicateurs qui affectent le même élément dans les deux Quantics, suivent la loi des multiplicateurs dans un Quantic préparé: ainsi comme cas particulier, si l'on introduit deux substitutions contraires, l'une dans un Quantic de la forme normale $\left(a, b, c, \ldots \nmid(x, y)^{i}\right.$, l'autre dans un Quantic où les éléments sont les mêmes mais dépourvus de tout multiplicateur numérique (c. à d. dans deux Quantics avec les mêmes éléments, l'une écrite selon la méthode ancienne, l'autre selon la méthode moderne) les deux substitutions induites sur les éléments seront contraires. A l'aide de cette remarque on évite l'inconvénient d'introduire des racines carrées qui doivent nécessairement disparaître dans les résultats.

Passons à l'application du théorème sur les substitutions contraires. Soit $F(a, b, c, \ldots: x, y)$ un covariant d'un Quantic ou d'un système de Quantics : écrivons comme auparavant $\dot{a}, \dot{b}, \dot{c}, \ldots$ pour $\frac{d}{d a}, \frac{d}{d b}, \frac{d}{d c}, \ldots ;$ je dis que $F(\dot{a}, \dot{b}, \dot{c}, \ldots: x, y)$ possédera toutes les propriétés d'un contravariant, c. à d. que si $G(a, b, c, \ldots: x, y)$ est un contravariant quelconque du même Quantic ou du même système, $F(\dot{a}, \dot{b}, \dot{c}, \ldots: x, y)$ appliqué comme opérateur à la forme $G(a, b, c, \ldots: x, y)$ conduira à un contravariant. Dans cet énoncé on suppose toutefois que les Quantics soient exprimés shacun dans leurs formes préparées ou bien (ce qui revient au même) que des deux formes $F$ et $G$ l'une appartienne à un système de Quantics pleins (c. à d. à éléments affectés de nombres binômes) et l'autre à un système de Quantics vides (c. à d. à éléments dépourvus de multiplicateurs binômes). Sous cette condition la forme $F * G$, c. à d. le résultat de l'opération du covariant $F$ sur le contravariant $G$, sera un contravariant du système auquel $G$ appartient. Si au contraire $F$ est un contravariant et $G$ un covariant le résultat $F \cdot G$ de l'opération de $F^{\prime}$ sur $G$ sera un covariant. Dans ce qui suit je supposerai pour plus de simplicité que les formes dont il est question soient présentées dans leur forme préparée.

Je vais passer maintenant à des générations de formes dérivées que l'on obtient, si dans la forme dérivée $F^{\prime}(a, b, c, \ldots: x, y)$, qui pourra être covariant ou contravariant, on remplace non seulement les éléments $a, b, c, \ldots$ par leurs inverses symboliques, c. à d. par $\dot{a}=\frac{d}{d a}, \dot{b}=\frac{d}{d b}, \dot{c}=\frac{d}{d c}, \ldots$, mais en même temps les variables $x, y$ par leurs inverses symboliques, c. à d. par

$$
\dot{x}=\frac{d}{d x}, \quad \dot{y}=\frac{d}{d y} .
$$


Soit $\Phi$ ce que devient $F$ après ce remplacement, de sorte que

$$
\Phi=F(\dot{a}, \dot{b}, \dot{c}, \ldots: \dot{x}, \dot{y}),
$$

et soit $G(a, b, c, \ldots: x, y)$ une seconde forme dérivée du même système, qui pourra être covariant ou contravariant; cela posé, suivant que le produit $F . G$ (c. à d. $F(a, b, c, \ldots: x, y)$ multiplié par $G(a, b, c, \ldots: x, y))$ est un covariant ou un contravariant, le résultat $\Phi \circledast G$ de l'opération de $\Phi$ sur $G$ sera également un covariant ou un contravariant.

Considérons encore l'opération $\Psi$ qui résulte d'une forme dérivée $F$ lorsque, sans altérer les éléments, on remplace seulement les variables $x, y$ par leurs inverses symboiliques $\dot{x}=\frac{d}{d x}, \dot{y}=\frac{d}{d y}$. Dans ce cas comme dans celui que nous avons considéré en premier lieu et dans lequel on remplaçait seulement les éléments (et non les variables) par leurs inverses symboliques, le caractère de $F$ est renversé, de covariant il devient contravariant et vice versâ. En un mot: une seule inversion symbolique renverse, deux inversions simultanées reproduisent le caractère de $F$. Ces propositions n'ont pas besoin d'être démontrées formellement, elles découlent comme conséquences des deux principes:

$1^{\circ}$. que la marche du mouvement d'un système quelconque de lettres et de leurs inverses symboliques est contraire,

$2^{\circ}$. que les mouvements induits dans les éléments* d'un Quantic préparé par deux mouvements contraires des variables sont eux-mêmes contraires.

Donnons le nom de différentiant-en- $x$ à une fonction $D^{\prime}$ des éléments d'un Quantic binaire ou d'un système de plusieurs Quantics binaires, qui ait la propriété de rester la même après la substitution de $x+h y$ au lieu de $x$, c. à d. qui dans la notation pleine d'éléments affectés de multiplicateurs binômes satisfasse à l'identité

$$
\Sigma(a \dot{b}+2 b \dot{c}+3 c \dot{d}+\ldots+i k \dot{l}) D^{\prime}=0 .
$$

De même soit ' $D$ un différentiant-en-y, c. à d. une fonction des éléments qui satisfasse à l'identité

$$
\Sigma\{i b \dot{a}+(i-1) \dot{c} \dot{b}+\ldots+l \dot{k}\}^{\prime} D=0 .
$$

Pour les Quantics préparés ces équations prennent la forme

(1) $\Sigma[\sqrt{ }(n) a \dot{b}+\sqrt{ }\{2(n-1)\} b \dot{c}+\ldots+\sqrt{ }\{2(n-1)\} h \dot{k}+\sqrt{ }(n) k \dot{l}] D^{\prime}=0$,

(2) $\Sigma[\sqrt{ }(n) b \dot{a}+\sqrt{ }\{2(n-1)\} c \dot{b}+\ldots+\sqrt{ }\{2(n-1)\} k \dot{h}+\sqrt{ }(n) l \dot{k}]^{\prime} D=0$.

On sait que $D^{\prime}$ sera toujours le coefficient de la plus haute puissance de $x$ dans quelque covariant du système et ' $D$ celui de la plus haute puissance de $y$

* De la combinaison de ces deux principes il résulte que le second principe peut être énoncé non seulement pour les éléments mais également pour leurs inverses symboliques. 
dans quelque contravariant; et puisque en substituant au lieu des éléments leurs inverses symboliques le résultat de son action sur le covariant sera en vertu de notre dernier théorème un covariant et le coefficient de la plus haute puissance en $x$ un différentiant-en- $x$, on en tire la conséquence que l'action d'un différentiant-en-y rendu opératif (par inversion symbolique) sur un différentiant-en- $x$ donnera naissance à un différentiant-en- $x$ : ce qui revient à dire que si $\dot{\Phi}$ est une fonction (des systèmes de $\dot{a}, \dot{b}, \dot{c}, \ldots$ ) qui satisfait à l'équation (2) quand on y remplace $a, b, c, \ldots \operatorname{par} \dot{a}, \dot{b}, \dot{c}, \ldots$ et $\dot{a}, \dot{b}, \dot{c}, \ldots \operatorname{par}$ $\frac{d}{d \dot{a}}, \frac{d}{d \dot{b}}, \frac{d}{d \dot{c}}, \ldots$ c. à d. par $\ddot{a}, \ddot{b}, \ddot{c}, \ldots$ et que si $D^{\prime}$ satisfait à l'équation (1), alors $\dot{\Phi} D^{\prime}$ doit satisfaire à la même équation (1).

Pour donner une démonstration indépendante de cette conclusion, je nommerai $\Omega^{\prime}$ l'opérateur qui reduit $D^{\prime}$ à zéro, ' $\Omega$ celui qui réduit $D$ à zéro. La démonstration restant essentiellement la même dans le cas d'un système et dans celui d'un seul Quantic, on se bornera pour plus de simplicité à ce dernier cas, ce qui permet de supprimer les signes de sommation $(\Sigma)$. Evidemment la proposition qu'on veut établir sera vraie si les deux opérations $\dot{\Phi} \Omega^{\prime}$ et $\Omega^{\prime} \dot{\Phi}$ que l'on obtient en appliquant l'opération $\dot{\Phi}$ et l'opération $\Omega^{\prime}$ l'une après l'autre dans un ordre différent, ne diffèrent pas entre elles, ou ce qui est la même chose, si $\dot{\Phi} \Omega^{\prime}$ et $\Omega^{\prime} \dot{\Phi}$ ne diffèrent pas en puissance opérative.

Or bornons-nous pour le moment à un seul terme quelconque, p.e. au terme $\lambda p \dot{q}$ contenu dans $\Omega^{\prime}$ ( $\lambda$ étant un nombre), et considérons la différence entre l'opération de $p \dot{q} \dot{\Phi}$ et de $\dot{\Phi} p \dot{q}$. Comme ce n'est que l'existence de $\dot{p}$ en $\dot{\Phi}$ qui produit cette différence, étudions l'effet de chaque terme $M \dot{p}^{i}$ séparément où $M$ ne contient pas $p$. D'après le théorème de Leibnitz la différence entre l'effet de $\dot{p}^{i}(p \dot{q})$ et de $p \dot{q}\left(\dot{p}^{i}\right)$ sera $\dot{q} i \dot{p}^{i-1}$, c. à d. $\dot{q} \frac{d}{d \dot{p}}\left(\dot{p}^{i}\right)$ ou bien $\ddot{q} \ddot{p}\left(\dot{p}^{i}\right)$.

Donc la valeur de la différence opérative entre $p \dot{q} \dot{\Phi}$ et $\dot{\Phi} p \dot{q}$ sera $\dot{q} \ddot{p} \dot{\Phi}$, et conséquemment la valeur totale de la différence entre $\Omega^{\prime} \dot{\Phi}$ et $\dot{\Phi} \Omega^{\prime}$ sera $\Sigma(\lambda \dot{q} \ddot{p} \dot{\Phi})$, c. à d. elle sera ce que $\Omega^{\prime}$ devient quand après avoir renversé l'ordre des lettres dans chaque conjonction $a \dot{b}, b \dot{c}, c \dot{d}$ qui s'y trouve, on remplace les lettres non-accentuées par les lettres une fois accentuées et ces dernières par les lettres deux fois accentuées, ce qui fait voir que la différence opérative entre $\dot{\Phi} \Omega^{\prime}$ et $\Omega^{\prime} \dot{\Phi}$ sera nulle, vu que par hypothèse $\dot{\Phi}(\dot{a}, \dot{b}, \dot{c}, \ldots)$ est un différentiant-en-y de l'expression dans laquelle sechange le Quantic donné (ou bien les Quantics simultanés donnés) quand on y remplace les éléments $a, b, c, \ldots$ par leurs inverses $\dot{a}, \dot{b}, \dot{c}, \ldots$, et que $\Omega^{\prime}$ se change en ' $\Omega$ quand on renverse l'ordre des éléments. J'ajouterai un seul exemple pour illustrer ce résultat, et pour éviter l'emploi des racines carrées je me servirai de la forme pleine pour les opérandes et de la forme vide pour les opérateurs.

S. III. 
Soit donné le Quantic $(x, y)^{3}$ et choisissons-en le discriminant

$$
a^{2} d^{2}+4 a c^{3}+4 d b^{3}-3 b^{2} c^{2}-6 a b c d .
$$

Differentiant par rapport à $a$ cet invariant que je regarde pour l'instant comme un différentiant-en-y, j'obtiens le nouveau différentiant-en-y

$$
a d^{2}-3 b c d+2 c^{3} \text {. }
$$

Pour obtenir l'opérateur qui y répond par rapport à la forme vide, il faut écrire $\frac{b}{3}, \frac{c}{3}$ au lieu de $b, c$, ce qui donne l'opérateur

$$
27 \dot{a} \dot{d}^{2}-9 \dot{b} \dot{c} \dot{d}+2 \dot{c}^{3}
$$

Appliquons cet opérateur au différentiant-en- $x$, que l'on obtient en multipliant le discriminant par $a c-b^{2}$ et qui est

$$
4 a^{2} c^{4}-7 a b^{2} c^{3}-\left(6 a^{2} b d+3 b^{4}\right) c^{2}+\left(a^{3} d^{2}+10 a b^{3} d\right) c-a^{2} b^{2} d^{2}-4 b^{5} d .
$$

Le résultat des différentiations indiquées sera

$$
192 a^{2} c-84 a b^{2}-270 a b^{2}+108 a^{2} c-108 a b^{2}+162 a^{2} c=462 a\left(a c-b^{2}\right),
$$

ce qui est évidemment un différentiant-en- $x$, comme il doit être. Passons rapidement à l'établissement des théorèmes analogues relatifs aux formes dérivées d'un nombre quelconque de variables.

La loi des mouvements contraires étant vraie pour les Quantics binaires dûment préparés, sera également vraie pour les Quantics ternaires pareillement préparés. Car soit $i$ le degré d'un Quantic dans ses variables $x, y, z$; qu'on le range suivant les puissances ascendantes de $z$, évidemment chacun des Quantics binaires qui multiplient ces puissances sera dûment préparé. Le premier aura pour son équipement numérique les racines carrées des nombres binômes de l'ordre $i$, le second les racines carrées des nombres binômes de l'ordre $i-1$ multipliés chacun par $\sqrt{ } i$, le troisième les racines carrées des nombres binômes de l'ordre $i-2$ multipliés chacun par $\sqrt{ } \frac{1}{2} i(i-1)$, et ainsi de suite. Or il est facile de voir comme auparavant que le théorème sera vrai pour des substitutions quelconques s'il est vrai pour les substitutions pour lesquelles le déterminant est l'unité, et chaque substitution de ce dernier genre peut être effectuée par une succession de substitutions simples de la forme $x+h y ; y+k z$, etc. Donc on n'a besoin que de démontrer le théorème pour une seule substitution de ce genre comme $x+h y$ : mais pour cette substitution, tous les Quantics en $x, y$ dont j'ai parlé étant dûment préparés, on a déjà démontré que le théorème est vrai. Donc le théorème est vrai pour chaque Quantic ternaire. De la même façon on passe des Quantics ternaires aux Quantics quaternaires et de même progressivement aux Quantics d'un nombre quelconque de variables. De plus il est facile de voir que la démonstration peut être étendue sans difficulté à des Quantics multipartites, c. à d. contenant un nombre quelconque de systèmes de 
variables: car chacun de ces systèmes étant assujetti à une substitution à part, le système des éléments subira une substitution composée des substitutions induites par chacune des substitutions partielles relatives à un système isolé de variables. De plus deux substitutions contraires appliquées à un quelconque des systèmes de variables induira deux substitutions contraires appliquées aux éléments.

Si pour donner plus de simplicité aux énoncés, on se borne au cas de Quantics unipartites, on peut résumer les conséquences qui découlent des principes établis en affirmant qu'une dérivée invariantive d'un système quelconque de Quantics unipartites préparés reste une dérivée invariantive, quand on substitue pour les variables ou pour les éléments ou pour les unes et les autres simultanément, leurs inverses symboliques avec la distinction que sous la première supposition le caractère est changé dans son opposé et sous la dernière il reste le même.-Dans mes premiers mémoires sur ce sujet dans le Quarterly Journal of Mathematics j’ai déjà donné substantiellement cette loi en me servant de la forme pleine pour les opérandes et de la forme vide pour les opérateurs-mais je crois que personne n'en a jamais donné la preuve.-C'est l'idée lumineuse et très-inattendue de la loi des mouvements contraires relative aux Quantics préparés qui simplifie la théorie et en rend la démonstration presque intuitive.

Cependant ce n'est que par exception qu'on doit se servir de la forme préparée pour désigner les Quantics.-Parmi les autres avantages de la notation ordinaire on peut citer la permanence de chaque expression d'un différentiant, c. à d. qu'un différentiant qui appartient à un Quantic d'un degré quelconque restera un différentiant de tout Quantic contenant le même nombre de variables d'un degré supérieur. Car soit

$$
\Omega=a \dot{b}+2 b \dot{c}+\ldots+i k \dot{l} \text { et } \Omega F(a, b, c, \ldots, l)=0,
$$

il est évident que si l'on augmente $\Omega$ par des termes additionnels

$$
(i+1) l \dot{m}+\text { etc. }
$$

et que l'on désigne par $\Omega_{1}$ l'opération $\Omega$ augmenté, on aura

$$
\Omega_{1} F(a, b, c, \ldots, l)=0 .
$$

Dans cette notation un covariant ou contravariant qui appartient à un Quantic quelconque donné, appartiendra donc également à tout autre Quantic composé du même nombre de variables et qui, en dépendant des mêmes éléments, s'élève pourtant à un degré supérieur*

* Il peut arriver qu'un différentiant qui est irréductible pour un degré donné de son Quantic cesse de l'être pour un degré supérieur. Cela a lieu, par exemple, dans le cas du discriminant de la forme binaire du troisième ou du cinquième degré (il va sans dire qu'en élevant le degré, on augmente en même temps le nombre des éléments). Il y a done des différentiants qui sont absolument irréductibles et d'autres qui ne le sont que conditionellement. Ainsi $a^{2} d-3 a b c+2 b^{3}$ 
De plus il y a dans cette notation des moyens qui permettent de donner à un différentiant unique la faculté de propager, pour ainsi dire, son espèce, sans agir sur une autre forme du même genre et sans en subir l'action.Voici un exemple de ce genre de propagation. Soit $F(a, b, c, \ldots, l)$ un différentiant-en- $x$ d'un Quantic binaire donné $\left(a, b, c, \ldots, l \gamma(x, y)^{j}\right.$, de l'ordre $j$ dans les éléments; remplaçons les éléments $a, b, c, \ldots, l$ de $F$ par $s_{0}, s_{1}, s_{2}, \ldots, s_{j}$; où $s_{q}$ signifie la somme des puissances $q^{\text {èmes }}$ des racines $\frac{x}{y}$ du Quantic donné; alors $\alpha^{\mu} F\left(s_{0}, s_{1}, s_{2}, \ldots, s_{j}\right)$ restera encore un différentiant-en- $x$ du même Quantic, $\mu$ étant un nombre égal à l'ordre du différentiant transformé. En effet les formules connues du calcul différentiel pour passer d'un système donné de variables indépendantes à un autre, étant appliquées à la transformation de l'expression $\frac{d}{d \alpha_{1}}+\frac{d}{d \alpha_{2}}+\ldots+\frac{d}{d \alpha_{j}}$, où $\alpha_{1}, \alpha_{2}, \ldots, \alpha_{j}$ désignent les valeurs de $\frac{x}{y}$ qui annulent le Quantic donné, cette expression se transformera dans l'une et l'autre des deux expressions

$$
-\frac{1}{a}[a \dot{b}+2 b \dot{c}+3 c \dot{d}+\ldots], \quad\left[s_{0} \dot{s}_{1}+2 s_{1} \dot{s}_{2}+3 s_{2} \dot{s}_{3}+\ldots\right] .
$$

Par conséquent l'identité $(a \dot{b}+2 b \dot{c}+\ldots) D=0$ étant satisfaite, l'identité corrélative $\left(s_{0} \dot{s}_{1}+2 s_{1} \dot{s}_{2}+\ldots\right) \Delta=0$ le sera également, $\Delta$ désignant la transformée de $D$ selon la règle donnée; et comme à chaque différentiant appartient un seul covariant dont il constitue un coefficient principal, on a le moyen de passer par une substitution facile d'un invariant ou covariant à un autre covariant qui sera en général d'un degré différent par rapport aux variables. Ainsi par exemple si l'on regarde l'invariant $a e-4 b d+3 c^{2}$ appartenant au Quantic $\left(a, b, c, d, e \gamma(x, y)^{4}\right.$ comme un différentiant-en- $x$, on voit que $\alpha, \beta, \gamma, \delta$ étant les quatre valeurs de $\frac{x}{y}$ qui annulent le Quantic donné,

$$
a^{4}\left\{\left(\alpha^{4}+\beta^{4}+\gamma^{4}+\delta^{4}\right)-4(\alpha+\beta+\gamma+\delta)\left(\alpha^{3}+\beta^{3}+\gamma^{3}+\delta^{3}\right)+3\left(\alpha^{2}+\beta^{2}+\gamma^{2}+\delta^{2}\right)^{2}\right\}
$$

sera aussi un différentiant-en- $x$ du Quantic donné. On vérifie aisément que cette expression est :

$$
a^{4}\left[\frac{1}{3}\left\{\Sigma(\alpha-\beta)^{2}\right\}^{2}-\frac{2}{3} \Sigma(\alpha \gamma-\beta \delta)^{2}\right]=-b\left(a c-b^{2}\right)^{2}-\frac{3}{2} a^{2}\left(a e-4 b d+3 c^{2}\right) ;
$$

ainsi l'invariant $a e-4 b d+3 c^{2}$ donne naissance au différentiant $a c-b^{2}$ dont le poids est égal à $\frac{2.4}{2}-2$ et qui par conséquent sert à déterminer un

appartient à la première catégorie, $a^{2} d^{2}+4 a c^{3}+4 d b^{3}-3 b^{2} c^{2}-6 a b c d$ à la seconde; car en l'attribuant au Quantic $\left(a, b, c, d, e \gamma(x, y)^{4}\right.$, il peut être exprimé sous la forme

$$
\left(a c-b^{2}\right)\left(a e-4 b d+3 c^{2}\right)-a\left(a c e-a d^{2}+2 b c d-c^{3}-b^{2} e\right),
$$

c. à $d$. il devient une fonction entière des quatre différentiants

$$
a, a c-b^{2}, \quad a e-4 b d+3 c^{2}, \quad a c e-a d^{2}+2 b c d-c^{3}-b^{2} e .
$$


covariant quadratique de l'ordre 2 dans les éléments. Ainsi un invariant a servi à produire un covariant. La double représentation des différentiants au moyen des éléments et au moyen des racines, fournit une démonstration d'un théorème assez important dans la théorie des partitions qu'il serait peut-être difficile d'établir par une démonstration directe. Voici en quoi consiste ce théorème. Désignons le nombre de manières de composer un nombre $n$ avec $j$ des nombres $0,1,2, \ldots, i \operatorname{par}(n: i, j)$ : on sait que $(n: i, j)=(n: j, i)$ et l'on voit sans peine que si

$$
m+\mu=\ddot{i j}, \quad(m: i, j)=(\mu: i, j) .
$$

Cela posé, le théorème en question affirme que pour les valeurs de $m$ qui n'excèdent pas $\frac{1}{2} i j,(m: i, j)$ peut être égal à $(m-1: i, j)$ ou plus grand que ce nombre, mais non plus petit que ce nombre, ou, ce qui revient au même, que si $m$ est plus grand que $\frac{1}{2} i j,(m: i, j)$ ne peut pas être plus grand que $(m-1: i, j)$. L'une de ces propositions implique l'autre en vertu de l'égalité $(m: i, j)=(i j-m: i, j)$. C'est la première que je veux établir et je l'établirai au moyen de la seconde. Cette démonstration étant accomplie je ferai une généralisation facile et du théorème et de la démonstration qui y conduit, pour en faire l'extension aux systèmes de couples $i, j$.

En vertu de l'équation $\Omega D=0$, on sait selon l'observation précieuse que M. Cayley a fait le premier, que le nombre de différentiants linéairement indépendants de l'ordre $j$ dans les éléments, qui appartiennent à un Quantic binaire donné du degré $i$, dont le poids par rapport à $x$ est $w$, doit être égal à $(w: i, j)-(w-1: i, j)$.

Sans même se servir de cette proposition qui est certainement vraie mais qui exige la vérification de l'indépendance des équations qu'on obtient en satisfaisant à l'identité $\Omega D=0$, on peut affirmer avec une certitude absolue que le nombre des différentiants dont il s'agit ne peut pas étre inférieur d $(w: i, j)-(w-1: i, j)$, ce qui suffit pour la démonstration proposée. Or je dis qu'il ne peut pas exister de différentiants pour lesquels $w$ est plus grand que $\frac{1}{2} \ddot{i j}$. Car en vertu de l'identité $\Omega=a \Sigma \frac{d}{d \alpha}$ un différentiant quelconque doit être de la forme $a^{j} \Sigma\left(\alpha-\alpha^{\prime}\right)\left(\alpha^{\prime \prime}-\alpha^{\prime \prime \prime}\right)\left(\alpha^{\mathrm{IV}}-\alpha^{\mathrm{V}}\right) \ldots$ où le nombre des facteurs est $w$ et chaque $\alpha$ une des $i$ valeurs de $\frac{x}{y}$ qui annulent le Quantic donné du degré $i$, bien entendu qu'il n'y a nulle restriction sur la répétition des mêmes racines. L'ordre de cette fonction symétrique relatif aux coefficients étant $j$, on en conclura d'après un théorème connu de l'algèbre ordinaire qu'aucune racine $\alpha$ ne peut se présenter plus de $j$ fois, mais dans chacun des $w$ facteurs il paraîtra deux lettres; donc le poids $w$ est la moitié du nombre total de ces apparitions. Or puisque nulle lettre ne paraît plus de $j$ ois, le nombre total de ces apparitions aura $i j$ pour son maximum et conséquemment la valeur maximum de $w$ est $\frac{1}{2} i j$, c. à d. qu'il n'existe pas de 
différentiants pour lesquels $w$ excède $\frac{1}{2} i j$; donc comme il existe toujours $(w: i, j)-(w-1: i, j)$ au moins (je dis au moins"pour ne pas m'appuyer sur la vérification de l'indépendance citée plus haut), il s'ensuit que, pour $w>\frac{1}{2} i j,(w: i, j)$ ne peut pas excéder $(w-1 ; i, j)$ et par conséquent que pour $w=\frac{1}{2} i j$ ou pour $w<\frac{1}{2} i j,(w: i, j)$ ne peut pas être plus petit que $(w-1: i, j)$, ce qu'il fallait démontrer. On peut étendre ce raisonnement en se fondant sur la proposition que pour un nombre quelconque de Quantics, p. e. pour deux Quantics $\left(a, b, c, \ldots, l \gamma(x, y)^{i},\left(a^{\prime}, b^{\prime}, \ldots, f^{\prime} \chi x, y\right)^{i^{\prime}}\right.$, le nombre des différentiants du poids $w$ en $x$, de l'ordre $j$ dans $a, b, c, \ldots$ et de l'ordre $j^{\prime}$ dans $a^{\prime}, b^{\prime}, \ldots$ a pour expression ou au moins pour valeur maximum* la différence entre deux dénumérants dont l'un est le nombre de solutions en nombres positifs entiers du système

$$
\begin{gathered}
x_{0}+x_{1}+\ldots+x_{i}=j, \quad y_{0}+y_{1}+\ldots+y_{i^{\prime}}=j^{\prime}, \\
x_{1}+2 x_{2}+\ldots+i x_{i}+y_{1}+2 y_{2}+\ldots+i^{\prime} y_{i^{\prime}}=w,
\end{gathered}
$$

et l'autre le dénumérant du système qui en résulte lorsqu'on y remplace $w$ par $w-1$. En suivant cette voie et après avoir démontré par la même méthode dont on s'est servi ci-dessus et à l'aide des fonctions symétriques des racines des deux Quantics donnés que la valeur maximum de $w$ est $\frac{i j+i^{\prime} j^{\prime}}{2}$, on arrivera à cette conclusion analogue que la valeur de la différence entre ces deux dénumérants ne peut jamais être négative, conclusion qui reste vraie en général. A ce résultat on peut donner l'énoncé remarquable: Que l'on développe suivant les puissances de $a, b, c, \ldots$ le produit d'un nombre quelconque de fonctions

$$
\begin{aligned}
& {\left[(1-a)(1-a t)\left(1-a t^{2}\right) \ldots\left(1-a t^{i}\right)\right]^{-1} \times} \\
& {\left[(1-b)(1-b t)\left(1-b t^{2}\right) \ldots\left(1-b t^{k}\right)\right]^{-1} \times} \\
& {\left[(1-c)(1-c t)\left(1-c t^{2}\right) \ldots\left(1-c t^{l}\right)\right]^{-1} \times}
\end{aligned}
$$

que l'on cherche dans ce développement la fonction de $t$ qui multiplie un produit quelconque donné de puissances de $a, b, c, \ldots$; cette fonction ordonné suivant les puissances ascendantes de $t$ présentera une série de coefficients numériques distribués symétriquement autour de son milieu et ayant des valeurs non décroissantes depuis l'une des extrémités jusqu'au terme unique ou jusqu'aux deux termes qui forment le milieu de la série.

L'importance de cette proposition pour la théorie des invariants consiste dans le fait qu'elle énonce et d'après lequel l'expression analytique du nombre des différentiants linéairement indépendants d'un système de

* Abstraction faite de l'indépendance non démontrée des équations données par l'application de l'opérateur $\left(a \frac{d}{d b}+2 b \frac{d}{d c}+\ldots\right)+\left(a^{\prime} \frac{d}{d b^{\prime}}+2 b^{\prime} \frac{d}{d c^{\prime}}+\ldots\right)$. 
Quantics binaires donné est toujours un nombre positif ou nul, pourvu que ces différentiants soient d'une forme concevable, c. à d. que le poids donné $w$ n'excède pas le maximum dont il est susceptible. Au contraire, pour les différentiants inconcevables, c. à d. dont le poids donné excède le maximum, l'expression analytique du nombre est toujours négatif ou zéro.

La loi de l'accroissement et du décroissement citée ci-dessus peut être exprimée au moyen d'une transformation facile à vérifier. En se bornant au cas d'un seul Quantic on a l'énoncé qu'en représentant par $\chi(\theta)$ un des deux produits

$$
\begin{aligned}
& \left(1-2 a \cos \theta+a^{2}\right)\left(1-2 a \cos 3 \theta+a^{2}\right) \ldots\left\{1-2 a \cos (2 q+1) \theta+a^{2}\right\}, \\
& (1-a)\left(1-2 a \cos 2 \theta+a^{2}\right)\left(1-2 a \cos 4 \theta+a^{2}\right) \ldots\left(1-2 a \cos 2 q \theta+a^{2}\right),
\end{aligned}
$$

la fraction $\frac{\sin \theta}{\chi(\theta)}$ développée selon les puissances positives de $a$ et les sinus des multiples de $\theta$ sera omnipositive, c. à d. ne contiendra que des coefficients numériques positifs.-La même conclusion aura lieu quand on multiplie ensemble plusieurs fonctions de l'une ou l'autre forme de $\chi$, savoir

$$
\chi(a, q, \theta) \chi\left(a^{\prime}, q^{\prime}, \theta\right) \ldots
$$

En désignant par $\Pi \chi(\theta)$ ce produit, le développement de $\frac{\sin \theta}{\Pi_{\chi}(\theta)}$ suivant les puissances de $a, a^{\prime}, a^{\prime \prime}, \ldots$ et leurs combinaisons et suivant les sinus des multiples de $\theta$ sera omnipositif.

Il paraît que ce théorème reste vrai quand on considère la fonction entière $\sin \theta \Pi \chi(\theta)$ au lieu de la fonction fractionnaire, mais je n'en possède point de preuve. Dans le cas simple de $\sin \theta \chi(\theta)$ cela reviendrait à l'énoncé que les coefficients des puissances de $t$ dans le développement de

$$
(1+a)(1+a t)\left(1+a t^{2}\right) \ldots\left(1+a t^{i}\right),
$$

qui forme évidemment une série symétrique, jouissent de la même propriété que les coefficients du développement de la fonction réciproque, c. à d. que les valeurs des coefficients peuvent augmenter ou rester stationnaires en passent de l'une ou l'autre extrémité de la série vers le milieu, mais qu'elles ne peuvent jamais décroître.-Il paraît qu'une proposition analogue peut être avancée pour le produit $\phi(1) \phi(t) \phi\left(t^{2}\right) \ldots \phi\left(t^{i}\right)$, où $\phi(x)$ signifie

$$
1+a x+a^{2} x^{2}+\ldots+a^{j} x^{j},
$$

et pour des formes encore plus générales. 
Dans les recherches précédents je suis tombé sur une démonstration exacte du théorème fondamental de la théorie des invariants, théorème qui a été accepté comme vrai par son illustre auteur M. Cayley sur la foi d'une induction à posteriori purement empirique et dont l'exactitude a été révoquée en doute par un écrivain distingué sur les formes binaires, apparemment en conséquence d'une méprise relative à l'explication donnée par M. Cayley sur la source de la conclusion erronée qu'il avait énoncée sur le nombre des invariants fondamentaux pour les degrés supérieurs.

On démontre facilement que si $D(w: i, j)$ est le nombre des différentiants linéairement indépendants* de l'ordre $j$, du poids $w$, et qui appartiennent à un Quantic binaire du degré $i$,

$$
D(w: i, j)=\text { ou }>(w: i, j)-(w-1: i, j),
$$

différence que je dénoterai désormais par $\Delta(w: i, j)$. Cette conclusion est une conséquence immédiate de l'identité $\Omega D=0, D$ étant un différentiant quelconque du Quantic $\left(a, b, c, \ldots, l \gamma(x, y)^{i}\right.$ et $\Omega$ l'opérateur

$$
a \delta_{b}+2 b \delta_{c}+3 c \delta_{d}+\ldots
$$

Mais pour établir le théorème en question, c. à d. l'équation

$$
D(w: i, j)=\Delta(w: i, j),
$$

il faudrait avoir prouvé l'indépendance de toutes les équations entre les constantes indéterminées, que l'identité $\Omega D=0$ fournit (en regardant $D$ comme une fonction composée des combinaisons des $a, b, c, \ldots$ multipliées chacune par une telle constante) — ce qui n'a jamais été fait et offre des difficultés presque insurmontables si l'on se propose de résoudre la question par escalade.-Je suivrai une marche différente-commençant par l'alternative d'égalité ou de supériorité entre $D$ et $\Delta$, je démontre que la dernière est inadmissible-l'indépendance dont j’ai parlé est donc une conséquence et non la clef de la démonstration.-Lorsqu'un opérateur quelconque $\Phi$ satisfait à l'équation $\Phi F^{\prime}=G$, je dirai dans ce qui suit que $\Phi$ transforme $F$ en $G$, et lorsqu'on a identiquement $\Phi F^{\prime}=0$, je dirai que $\Phi$ annule $F$.

Je remarque que $D(0: i, j)=1$ parce que dans tous les cas il y a un seul différentiant-en- $x$ libre du poids zéro, à savoir une puissance de $a$ : d'autre part $(0: i, j)$, c. à d. le nombre de manières de composer zéro avec $0,1,2,3, \ldots, i$ prises $j$ à $j$, est aussi $=1$, par conséquent la relation $D(w: i, j) \equiv \Delta(w: i, j)$ fournit

$$
D(w: i, j)+D(w-1: i, j)+D(w-2: i, j)+\ldots+D(0: i, j) \bar{s}(w: i, j),
$$

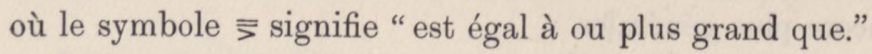

* Pour plus de commodité je dirai différentiants libres au lieu de différentiants linéairement indépendants. 
De plus on aura

$$
\begin{gathered}
D(w: i, j)+2 D(w-1: i, j)+3 D(w-2: i, j)+\ldots \\
\quad(w: i, j)+(w-1: i, j)+(w-2: i, j)+\ldots,
\end{gathered}
$$

ce qui est vrai pour toutes les valeurs de $w$. Je supposerai à présent que $w$ ait la valeur $\frac{1}{2} i j$ pour $i j$ pair et la valeur $\frac{1}{2}(i j-1)$ pour $i j$ impair. Dans ce cas la somme qui forme la seconde partie de la dernière relation devient évidemment égale au nombre des combinaisons $j$ à $j$ (avec répétitions) formées des $(i+1)$ chiffres $0,1,2,3, \ldots, i$, et assujetties à la restriction que la somme des chiffres d'une combinaison n'excède pas $\frac{1}{2} i j$ : nombre de combinaisons que je dénoterai par $P(i, j)$.

Remplaçons chaque différentiant qui fait partie du groupe dont le nombre est $D(w: i, j)$, de même du groupe dont le nombre est $D(w-1: i, j)$, etc. par le covariant qui y correspond.-Le degré de ces covariants par rapport aux variables étant, pour une valeur quelconque de $w, \ddot{j}-2 w$, les degrés des covariants dans les groupes successifs seront $1,3,5, \ldots$ dans le cas de $i j$ impair, et $0,2,4, \ldots$ dans le cas de $\ddot{i j}$ pair. Imaginons que chaque coefficient de chacun de ces covariants soit représenté par une dame d'un damier, qu'on se borne à prendre le premier coefficient des covariants du premier groupe, les deux premiers coefficients des covariants du second groupe, les trois premiers du troisième groupe, etc., on peut alors former un triangle rectangulaire de piles des dames. La pile au sommet contiendra $D(w: i, j)$, les deux piles qui suivent $D(w-1: i, j)$, les trois piles qui suivent $D(w-2: i, j)$ chacune, et ainsi de suite. Le nombre total des dames sera la fonction qui est $\equiv P(i, j)$.

Pour donner plus de précision à cette image je remarque que les dames dans la première colonne verticale représentent des différentiants et que chaque pile à la base se réduit nécessairement à une seul dame, dont la partie essentielle (abstraction faite de la partie numérique qui n'a pas d'influence sur le raisonnement et que je négligerai dans tout ce qui suit) n'est autre chose qu'un coefficient du Quantic du degré $i$ élevé à la puissance $j$.

Remarquons que, d'après une propriété bien connue des covariants, chaque quantité dans la première colonne sera annulée par $\Omega$, dans la seconde par $(\Omega)^{2}$, en général dans la $q^{\text {eme }}$ par $(\Omega)^{q}$ et que l'opérateur $(\Omega)^{q-1}$ appliqué à un terme de la $q^{\text {ème }}$ colonne produit le différentiant qui se trouve à la première place de la même horizontale avec ce terme.

Remarquons encore qu'en avançant de gauche à droite dans la même horizontale, les poids des quantités augmentent d'une unité de l'une à l'autre, qu'au contraire, en avançant de haut en bas dans la même verticale, les poids des quantités diminuent d'une unité de l'une à l'autre, de sorte 
que dans une ligne diagonale descendante (de gauche à droite), ou ce qui est la même chose, parallèle à l'hypoténuse, tous les termes sont de poids égal.

Or j'affirme que nulle liaison linéaire ne peut exister entre les quantités du triangle en question. Evidemment ce n'est qu'entre les quantités isobariques qu'une telle liaison serait imaginable. Prenons une ligne quelconque parallèle à l'hypoténuse ou bien l'hypoténuse elle-même.

$1^{\circ}$. Je dis que nulle équation linéaire ne peut lier des quantités qui se trouvent exclusivement dans une seule pile. Car si cette pile se trouvait dans la $q^{\text {ème }}$ colonne, en vertu du fait que l'opérateur $(\Omega)^{q-1}$ fait naître de chacune d'elle le différentiant qui se trouve à la première place de la même ligne horizontale, la liaison supposée subsisterait encore entre des différentiants d'une même pile, ce qui est contraire à l'hypothèse de la construction.

$2^{\circ}$. Je dis que nulle équation linéaire ne peut lier les quantités qui se trouvent dans des piles distinctes. En effet, supposons donnée une relation de ce genre, d'après la condition du poids égal il ne peut y avoir dans chaque colonne qu'une seule pile comprenant des quantités qui entrent dans l'équation supposée. Soit $q$ le rang de la colonne la plus avancée qui renferme une pile comprenant des quantités liées entre elles par l'équation linéaire. L'opérateur $\Omega$, appliqué au premier membre de l'équation qui exprime cette liaison un nombre de fois inférieur à $q-1$, produira une équation d'une forme analogue. Mais lorsqu'on applique l'opérateur $(\Omega)^{q-1}$, toutes les quantités comprises dans des colonnes d'un rang inférieur à $q$ seront annulées, tandis que celles qui sont comprises dans la pile de la $q^{\text {ème }}$ colonne seront transformées en des différentiants appartenant à la même ligne, c. à d. qu'il y aurait une liaison linéaire entre les différentiants d'une même pile, ce qui est contraire à l'hypothèse de la construction.

On a donc démontré que nulle équation linéaire ne subsiste entre les quantités du triangle.-De plus il est évident que le poids d'un coefficient quelconque qui se trouve dans le triangle ne peut excéder $\frac{1}{2} i j$. Donc les quantités comprises dans le triangle sont des fonctions linéaires et homogènes sans liaison linéaire entre elles de $P(i, j)$ quantités. Donc le nombre de ces quantités ne peut pas excéder $P(i, j)$, c. à d. que

$$
D(w: i, j)+2 D(w-1: i, j)+3 D(w-2: i, j)+\ldots
$$

ne peut pas excéder $P(i, j)$.

Mais si dans une seule des relations $D(w: i, j) \overline{>}(w: i, j)$, pour des valeurs de $w$ quelconques, le signe applicable était $>$ et non $=$, la somme en question serait $>P(i, j)$. 
Donc on a toujours $D(w: i, j)=\Delta(w: i, j)$, ce qu'il fallait démontrer. Comme corollaire il s'ensuit que l'indépendance des équations données par l'identité $\Omega D=0$ est établie. Précisément la même méthode peut être suivie pour démontrer l'égalité

$$
D\left(w: i, j: i^{\prime}, j^{\prime}: \text { etc. }\right)=\Delta\left(w: i, j: i^{\prime}, j^{\prime}: \text { etc. }\right)
$$

où $D$ dénote le nombre des différentiants libres d'un système de Quantics binaires, $i, i^{\prime}, \ldots$ désignant les degrés des Quantics, $j, j^{\prime}, \ldots$ l'ordre des différentiants par rapport aux coefficients de chacun des Quantics, et où $\Delta$ dénote la différence entre deux dénumérants, l'un désignant le nombre des solutions en nombres entiers et positifs du système des équations simultanées

$$
\begin{gathered}
x_{0}+x_{1}+x_{2}+\ldots+x_{i}=j, \quad x_{0}^{\prime}+x_{1}^{\prime}+\ldots+x_{i^{\prime}}^{\prime}=j^{\prime}, \text { etc. } \\
x_{1}+2 x_{2}+\ldots+i x_{i}+x_{1}^{\prime}+2 x_{2}^{\prime}+\ldots+i^{\prime} x_{i^{\prime}}^{\prime}+\ldots=w,
\end{gathered}
$$

et l'autre le dénumérant du système qui en résulte lorsqu'on y remplace $w$ par $w-1$.

Un autre corollaire que le théorème contient comme cas particulier est la proposition déjà démontrée, que $\Delta(w: i, j)$ ne peut jamais devenir négatif pour des valeurs de $w$ qui n'excèdent pas $\frac{1}{2} i j$. En effet si cette assertion n'était pas vraie, il devrait exister une valeur de $w$ qui n'excède pas $\frac{1}{2} i j$ et pour laquelle $D(w: i, j)>\Delta(w: i, j)$, ce qui a été prouvé impossible.

En dernier lieu je remarque qu'en démontrant inadmissible le signe de supériorité, on a établi pour $w=\frac{1}{2} \ddot{i j}$ quand $i j$ est pair et pour $w=\frac{1}{2}(i j-1)$ quand $i j$ est impair, l'équation

$$
D(w: i, j)+2 D(w-1: i, j)+3 D(w-2: i, j)+\ldots=P(i, j) .
$$

Soit $i j$ impair, en vertu de l'équation $(x: i, j)=(i j-x: i, j)$ le nombre $P(i, j)$ sera évidemment la moitié du nombre total des combinaisons $j$ à $j$ des $i+1$ éléments $0,1,2,3, \ldots, i$. Donc pour $i j$ impair $P(i, j)=\frac{1}{2} \frac{\Pi(i+j)}{\Pi i \Pi j}$.

Soit au contraire $i j$ pair, on aura

$$
\begin{aligned}
P(i, j) & =\frac{1}{2}\left\{\frac{\Pi(i+j)}{\Pi i \Pi j}+(w: i, j)\right\} \\
& =\frac{1}{2} \frac{\Pi(i+j)}{\Pi i \Pi j}+\frac{1}{2}\{D(w: i, j)+D(w-1: i, j)+D(w-2: i, j)+\ldots\} .
\end{aligned}
$$

Le degré des covariants qui correspondent un à un aux différentiants dont le nombre est $D(x: i, j)$ étant $i j-2 x$, on peut substituer pour $D(x: i, j)$ le nombre $K(i, j: i j-2 x)$ où $i$ est le degré du Quantic donné, $j$ l'ordre par rapport aux coefficients, $i j-2 x$ le degré relatif aux variables des covariants dont $K$ exprime le nombre total. 
Par conséquent quand ij est impair, on aura et quand $\ddot{i j}$ est pair

$$
2 K(i, j: 1)+4 K(i, j: 3)+6 K(i, j: 5)+\ldots=\frac{\Pi(i+j)}{\Pi i \Pi j}
$$

$$
K(i, j: 0)+3 K(i, j: 2)+5 K(i, j: 4)+\ldots=\frac{\Pi(i+j)}{\Pi i \Pi j} .
$$

En remarquant que pour $\ddot{\imath}$ impair il n'existe pas de covariants de degré pair, et pour ij pair il n'en existe pas de degré impair, on peut réunir ces deux formules dans une seule formule remarquable, qui assujettit les quantités transcendantes $K$ à une loi algébrique et qui pourrait même être très-utile dans certains cas comme formule de vérification:

$$
K(i, j: 0)+2 K(i, j: 1)+3 K(i, j: 2)+\ldots=\frac{\Pi(i+j)}{\Pi i \Pi j} .
$$

J'en donnerai quelques exemples.

Soit

$$
i=4, \quad j=2 .
$$

On trouve

$$
\begin{gathered}
K(4,2: 0)=1 ; K(4,2: 2)=0 ; K(4,2: 4)=1 ; K(4,2: 6)=0 ; \\
K(4,2: 8)=1
\end{gathered}
$$

et de là

$$
1+5+9=15=\frac{\Pi 6}{\Pi 2 \Pi 4} \text {. }
$$

Soit

$$
i=3, \quad j=3 .
$$

En se rappelant l'échelle fondamentale pour les cubiques

$$
3.1,4.0,2.2, \quad 3.3
$$

on trouve

$$
\begin{gathered}
K(3,3: 1)=0, \quad K(3,3: 3)=1, \quad K(3,3: 5)=1, \quad K(3,3: 7)=0, \\
K(3,3: 9)=1
\end{gathered}
$$

et de là

$$
4+6+10=20=\frac{\Pi 6}{\Pi 3 \Pi 3} \text {. }
$$

Soit

$$
i=3, \quad j=4 \text {. }
$$

On trouve

$$
\begin{gathered}
K(3,4: 0)=1, K(3,4: 2)=0, K(3,4: 4)=1, \quad K(3,4: 6)=1, \\
K(3,4: 8)=1, K(3,4: 10)=0, K(3,4: 12)=1
\end{gathered}
$$

et de là

$$
1+5+7+9+13=35=\frac{\Pi 7}{\Pi 3 \Pi 4} .
$$

Le théorème que j'ai vérifié par ces exemples peut être résumé dans les termes suivants. Chaque covariant d'un ordre donné $j$ par rapport aux coefficients d'un Quantic binaire de degré donné $i$, étant répété autant de fois qu'il y a de chiffres dans la série qui commence par zéro et se termine 
par le degré du covariant, relatif aux variables qui y entrent, le nombre total de ces expressions, chacune comptée autant de fois qu'elle est répétée, est égal au nombre binôme symétrique par rapport aux nombres $i$ et $j$, c. à d. égal à $\frac{\Pi(i+j)}{\Pi i \Pi j}$.

La règle des nombres binômes s'applique avec une modification légère au cas de plusieurs Quantics binaires de degrés donnés et de covariants d'ordres donnés relatifs aux coefficients de cẹs Quantics. Dans ce cas général on substituera au nombre binôme unique qui se présente dans le cas d'un seul Quantic, le produit de plusieurs nombres binômes dont chacun est symétrique par rapport au degré $i$ de l'un des Quantics et à l'ordre $j$ du covariant relatif aux coefficients du même Quantic.

Considérons comme exemple le cas de deux quadratiques binaires. Dans ce cas qui correspond à $i=2, i^{\prime}=2$ il y a trois covariants de l'ordre $j=1$ par rapport aux coefficients de chacune, savoir:

$1^{\circ}$ le produit des deux Quantics,

$2^{\circ}$ leur Hessien,

$3^{\circ}$ leur Connectif.

Les degrés de ces trois expressions relatifs aux variables étant respectivement $4,2,0$, on aura

$$
5+3+1=\left(\frac{\Pi 3}{\Pi 1 \Pi 2}\right)^{2}=9,
$$

ce qui s'accorde avec la règle énoncée ci-dessus.

A l'énumération que j'ai faite des propriétés essentielles du triangle de piles, j'ajoute la remarque que le poids maximum d'une quelconque des quantités qui s'y trouvent, est évidemment celui de la quantité qui appartient à l'hypoténuse et se trouve au sommet du triangle. Ce poids maximum est $\frac{1}{2} i j$ ou $\frac{1}{2}(\ddot{i j}-1)$ et par conséquent n'excède jamais $\frac{1}{2} i j$. C'est ainsi qu'on voit que les quantités comprises dans le triangle ne sont autre chose que des fonctions linéaires des combinaisons de l'ordre $j$ par rapport aux coefficients du Quantic proposé, combinaisons dont le nombre est $P(i, j)$.

Postscriptum 1. La démonstration donnée du théorème fondamental $D(w: i, j)=\Delta(w: i, j)$ peut être abrégée et simplifiée comme il suit.

$\mathrm{Au}$ lieu de se servir de la condition

$$
D .(w: i, j)+2 D(w-1: i, j)+\ldots>P(i, j)
$$

il suffit de considérer l'équation préalable

$$
\Sigma D(w: i, j)=(w: i, j) \text {. }
$$

Pour un différentiant quelconque que je désignerai par $[w-\delta]$ et dont le poids soit $w-\delta$ substituons l'expression $(' \Omega)^{\delta}[w-\delta]$, expression qui résulte 
de $[w-\delta]$ en $\mathbf{y}$ appliquant l'opérateur* $(' \Omega)^{\delta}$ et qui jouit de la propriété qu'en opérant sur elle avec $\left(\Omega^{\prime}\right)^{\delta}$, le résultat est un multiple numérique de $[w-\delta]$. Toutes les expressions ainsi obtenues seront du même poids $w$ et par conséquent des fonctions linéaires des $(w: i, j)$ combinaisons qui sont du poids $w$ et de l'ordre $j$ dans les $i+1$ coefficients du Quantic donné.

On démontre comme auparavant que ces expressions sont linéairement indépendantes entre elles et que par conséquent leur nombre ne peut pas excéder $(w: i, j)$; donc leur nombre est égal à $(w: i, j)$, et la proposition est établie.

Pour démontrer que $\left(\Omega^{\prime}\right)^{\delta}(' \Omega)^{\delta}[w-\delta]$ est, à un facteur numérique près, égal à $[w-\delta]$, on n'a pas besoin de sortir de la sphère des différentiants et de faire appel aux propriétés des covariants. On établit aisément que pour une quantité quelconque $D$ du poids $w$ et de l'ordre $j$ dans les coefficients d'un Quantic binaire du degré $i$, on aura

$$
\left(\Omega^{\prime \prime} \Omega-\Omega^{\prime} \Omega \Omega^{\prime}\right) D=(i j-2 w) D .
$$

En partant de là et supposant que $\Omega^{\prime} D=0$, on trouve par une induction algébrique facile que

$$
\left(\Omega^{\prime}\right)^{k}(' \Omega)^{k} D=1.2 \ldots k(i j-w)(i j-w-1) \ldots(i j-w-k+1) . D,
$$

où le facteur numérique ne s'évanouit que lorsque $k$ est plus grand que $i j-w$.

Considérons le système complet des expressions

$$
[w-\delta], \quad ' \Omega[w-\delta], \quad(' \Omega)^{2}[w-\delta], \ldots, \quad(' \Omega)^{i j-2 w}[w-\delta],
$$

dont la dernière, qui résulte de l'opération ' $\Omega$ répétée $i j-2 w$ fois, se réduit à un différentiant-en- $y$, tandis que les suivantes produites par la même opération répétée $i j-2 w+1$ ou un plus grand nombre de fois s'évanouissent identiquement.

En représentant toujours par des piles l'ensemble de toutes les expressions $\left({ }^{\prime} \Omega\right)^{m}[w-\delta]$ pour les mêmes valeurs de $m$ et de $\delta$, distinguant les deux cas de $i j$ pair ou impair, et commençant par la plus grande valeur de $w$ qui est $\frac{1}{2} i j$ pour $i j$ pair et $\frac{1}{2}(i j-1)$ pour $i j$ impair, on arrivera aux deux tableaux $†$ suivants de points, qui donnent une image du système en question de piles

$$
\text { pour ij impair }
$$

pour $i j$ pair

* Le signe $(' \Omega)^{\delta}$ exprime l'opération ' $\Omega$ répétée $\delta$ fois.

† Le point au sommet du premier tableau correspond à des invariants, les points à gauche se rapportent aux différentiants-en- $x$, ceux à droite aux différentiants-en- $y$, ceux de la base aux coefficients successifs du Quantic élevé à la puissance $j$ (pour l'un et l'autre des deux tableaux). 
Prenons l'ensemble de toutes les combinaisons des coefficients du Quantic proposé du degré $i$, qui sont de l'ordre $j$ dans les coefficients et des poids $0,1,2, \ldots, i j$ quant à $x$, alors, dans l'un cas comme dans l'autre, les quantités qui se trouvent dans chaque colonne verticale, seront du même nombre que l'ensemble correspondant des combinaisons des coefficients, elles seront en même temps des fonctions homogènes et linéaires des combinaisons qui $\mathrm{J}$ appartiennent.

Les piles qui se trouvent dans une ligne horizontale quelconque peuvent se réduire à une seule quantité, cas qui se présente toujours pour la dernière ligne horizontale: elles peuvent même s'évanouir identiquement, ce qui arrive pour certaines valeurs de $w, i, j$ pour lesquelles il n'existe point de différentiant.

Postscriptum 2. Je suppléerai dans ce qui suit à une lacune qui se trouve dans les recherches précédentes, en donnant la démonstration de la proposition suivante :

Dans un Quantic préparé les inverses symboliques des éléments subissent par une substitution quelconque des variables une substitution induite qui est identique avec celle que les éléments eux-mêmes subiraient par la substitution contraire des variables.

Les mêmes raisonnements dont on s'est déjà servi plusieurs fois, font voir que pour la démonstration générale de cette proposition il suffit de la vérifier dans le cas spécial dans lequel le Quantic est binaire et $x+\epsilon y$ la valeur que l'on substitue pour $x, \epsilon$ étant infiniment petit. Soit $i$ le degré du Quantic donné, soient $a, b, c, \ldots, h, k, l$ ses éléments et

$$
1, \quad \sqrt{ } i, \quad \sqrt{ }\left\{\frac{i(i-1)}{2}\right\}, \ldots, \quad \sqrt{ } i, \quad 1,
$$

les multiplicateurs numériques des éléments, soient $a^{\prime}, b^{\prime}, c^{\prime}, \ldots$ les valeurs dans lesquelles se changent les éléments donnés après la substitution de $x+\epsilon y$ au lieu de $x, \epsilon$ étant infiniment petit; cela posé et en faisant

$$
\lambda=\sqrt{ } i, \quad \mu=\sqrt{ }\{2(i-1)\}, \quad \nu=\sqrt{ }\{3(i-2)\}, \quad \cdots
$$

les nouveaux éléments et les éléments primitifs s'exprimeront les uns par les autres au moyen des relations linéaires

$$
\begin{aligned}
& a^{\prime}=a, \quad b^{\prime}=b+\lambda \epsilon a, \quad c^{\prime}=c+\mu \epsilon b, \quad \ldots, \quad k^{\prime}=k+\mu \epsilon h, \quad l^{\prime}=l+\lambda \epsilon k, \\
& a=a^{\prime}, \quad b=b^{\prime}-\lambda \epsilon a^{\prime}, \quad c=c^{\prime}-\mu \epsilon b^{\prime}, \quad \ldots, \quad k=k^{\prime}-\mu \epsilon h^{\prime}, \quad l=l^{\prime}-\lambda \epsilon k^{\prime} .
\end{aligned}
$$

D'autre part les inverses symboliques de ces deux systèmes d'éléments

$$
\begin{array}{cc}
\dot{a}=\frac{d}{d a}, & \dot{b}=\frac{d}{d b}, \ldots \\
\dot{a}^{\prime}=\frac{d}{d a^{\prime}}, & \dot{b}^{\prime}=\frac{d}{d b^{\prime}}, \ldots
\end{array}
$$


satisfont aux équations

$$
\begin{aligned}
& \dot{a}^{\prime}=\dot{a} \frac{d a}{d a^{\prime}}+\dot{b} \frac{d b}{d a^{\prime}}=\dot{a}-\lambda \epsilon \dot{b} \\
& \dot{b}^{\prime}=\dot{b} \frac{d b}{d b^{\prime}}+\dot{c} \frac{d c}{d b^{\prime}}=\dot{b}-\mu \epsilon \dot{c} \\
& \vdots \\
& \dot{k}^{\prime}=\dot{k} \frac{d k}{d k^{\prime}}+i \frac{d l}{d k^{\prime}}= \\
& \quad k-\lambda \epsilon \dot{l} \\
& \dot{l}^{\prime}=\dot{l} \frac{d l}{d l^{\prime}} \quad=i
\end{aligned}
$$

ce qui fait voir que la substitution des inverses symboliques $a, \dot{b}, \ldots, \dot{i}$ induite par la substitution de $x+\epsilon y$ au lieu de $x, y$ restant inaltéré, est précisément la même que la substitution contraire de $y-\epsilon x$ au lieu de $y, x$ restant inaltéré, induirait dans les éléments mêmes $a, b, \ldots, l$.

Je terminerai ces additions par l'énoncé d'un théorème général sur les formes invariantives dérivées qui montre d'une manière frappante le parti avantageux que l'on tire de la forme préparée sous laquelle je présente les Quantics.

Soit $F^{\prime}(a, b, c, \ldots: x, y, \ldots)$ un contravariant et $\Phi(a, b, c, \ldots: x, y, \ldots)$ un covariant du même Quantic donné; on connaît depuis longtemps le théorème que la nouvelle forme

$$
F\left(a, b, c, \ldots: \frac{d \Phi}{d x}, \frac{d \Phi}{d y}, \ldots\right)
$$

est un covariant du même Quantic. Or j'ajoute que si le Quantic proposé est présenté dans la forme préparée, alors la nouvelle forme

$$
F\left(\frac{d \Phi}{d a}, \frac{d \Phi}{d b}, \frac{d \Phi}{d c}, \ldots: x, y, \ldots\right)
$$

sera également un covariant du même Quantic. Si le Quantic proposé est présenté dans la forme ordinaire (pleine), cette dernière expression se change en

$$
F\left(\frac{1}{m} \frac{d \Phi}{d a}, \frac{1}{n} \frac{d \Phi}{d b}, \frac{1}{p} \frac{d \Phi}{d c}, \ldots: x, y, \ldots\right),
$$

$m, n, p, \ldots$ désignant les nombres binômes ou polynômes qui multiplient les éléments $a, b, c, \ldots$, elle se change au contraire en

$$
F\left(m \frac{d \Phi}{d a}, n \frac{d \Phi}{d b}, p \frac{d \Phi}{d c}, \ldots: x, y, \ldots\right),
$$

si le Quantic est présenté dans la forme vide. La démonstration de ce théorème se fait immédiatement à l'aide des principes exposés dans ce mémoire. 